\title{
Investigation of the Accuracy of the Elastic Settlement Estimation Methods and the Extrapolation Techniques of Plate Load Test Results
}

\author{
H. S. Thilakasiri and S. M. Priyantha
}

Abstract: Accurate estimation of the settlement of shallow foundations is an important aspect in the design of such foundations. Most often elastic settlement estimation methods are used in the estimation of settlement of shallow foundations. In addition, plate load tests are also performed to estimate the settlement of shallow foundations. Since a smaller size plate is used in the plate load test, the settlement obtained from such tests should be extrapolated to the actual size footings. Even though there are large number of settlement estimation methods and extrapolation techniques for plate load test results, the verification studies of the accuracy of the methods are very rare. It is very often observed that the settlement of shallow foundations are estimated based on the plate load tests carried out in layered soil medium, where a stiff fill soil layer overlying a soft soil. Extrapolation techniques of the plate load test results are highly questionable under such situations.

A finite element model was developed using the software called PLAXIS to simulate the settlement of footings. The developed model was used to model some case studies presented in the literature to investigate the accuracy of the model predictions. Thereafter, parametric studies were carried out to investigate the accuracy of: (i) commonly used clastic settlement estimation methods; (ii) the commonly used extrapolation techniques in the homogeneous subsurface conditions; and (iii) the commonly used extrapolation techniques in the two layered medium with a hard layer overlying a soft layer.

Keywords: Plate load test, shallow foundation, settlement

\section{Introduction}

Shallow foundations are the most common type of foundations used to support the structures due to the low cost and the simplicity of construction compared to pile foundations. Most of the shallow foundations are constructed without any geotechnical design inputs and, even if geotechnical designs are carried out, wrong assumptions and unreliable engineering judgments are used in the process. As a results, the percentage of failures of shallow foundations are much higher than the failures of deep foundations. It is absolutely critical to select the appropriate testing methodologies, correct interpretation methods and reliable design approach in the design of shallow foundations.

Failure of a foundation occurs mainly due to two different reasons: shear failure of the medium surrounding the foundation; and excessive settlement of the foundation under the working conditions. It is the responsibility of the designer to propose a foundation having a reasonable factor of safety against shear failure and the settlement under the working load within tolerable limits to the structure supported by the foundation. Moreover, it is observed that most of the failures of shallow foundations are due to excessive settlement of the foundation than due to shear failure. In this context, the designer should be in a position to estimate the settlement of the foundation under the action of the working load.

Settlement of foundations may be due to elastic (or immediate settlement), primary consolidation settlement and secondary consolidation settlement. It is intended to investigate the accuracy of the different elastic settlement estimation methods of shallow foundations in comparison to the predictions of the same from the finite element methods (FEM).

\footnotetext{
Eng. (Dr.) H. S. Thilakasiri, C. Eng., ME (Sri Lanka), Int. P. Eng(SL), B.Sc. Eng. (Hons) (Moratuwa), MSc. (London), DIC, PhD (USA), Senior Lecturer in

Engineering, Department of Civil Engineering, University of Moraluwa.

Eng. S. M. Priyantha, C. Eng., MIE (Sri Lanka), B. Sc. Eng. (Peradentya).
} 
As the measured settlement of full scale shallow foundations are costly, the accuracy of the FEM model used was verified using the load - settlement behaviour of footings reported in the literature.

In this study, the accuracy of three commonly used settlement estimation methods in comparison to the settlement predicted by the developed finite element model, are investigated. Furthermore, the effect of the friction angle on the settlement

Behaviour of the shallow foundations is also investigated.

The most accurate ultimate carrying capacity and settlement of the foundations can be estimated by loading a prototype foundation. However, such testing is very expensive, time consuming and most often not practically feasible. Therefore, a model of the actual foundation, very often a small size metal plate, is loaded and the results obtained are extrapolated to estimate the ultimate carrying capacity and the settlement of the prototype foundations. This method is commonly referred as the plate load test and used frequently in the design of shallow foundations. However, the accuracy of the estimated behavior of the prototype foundation depends very much on the accuracy of the extrapolation technique and its applicability to the given subsurface condition.

Plate load test is also used to obtain the modulus of subgrade reaction, a very important soil parameter in the design of raft foundations and combined footings. The modulus of subgrade reaction, defined, as the ratio between the stress applied on the soil and the resulting settlement, is not a material property. As the settlement of the shallow foundation depends on the width and the depth below the ground surface, the settlement obtained from the plate load test should be extrapolated to the size of the raft or the combined footing to estimate the modulus of subgrade reaction applicable to such foundations. Therefore, the settlement of the plate corresponding to the applied stress should be extrapolated before estimating the modulus of subgrade reaction for large foundations.

From the above discussion it is clear that the use of an accurate and reliable technique for extrapolation of the settlement of plate load tests is of vital importance in many geotechnical engineering applications. Therefore, in this research study, the accuracy of the settlement extrapolation techniques for plate load test results under different subsurface conditions are also investigated using the developed FEM model.

\section{Settlement Estimation of Shallow Foundations}

Settlement of shallow foundations may be obtained using elastic settlement estimation methods; empirical correlations based on insitu test results; and from the results of the plate load tests.

\subsection{Settlement estimation using elastic methods}

Even though there are large number of settlement estimation methods, three of the widely used methods given below are considered in the present study. All the elastic settlement estimation methods assume that the foundation is resting on an isotropic, homogeneous linear elastic soil medium. It should be noted here that due to the limitation of the space, the readers are referred to relevant literature for the determination of the factors relevant to different methods.

\subsubsection{Method proposed by Janbu (Bowels, 1988)}

Settlement $(\rho)$ is estimated using the Equation [1] given below.

$\rho=q \mu_{0} \mu_{1} B\left(\frac{1-\mu^{2}}{E_{s}}\right)$

Where

q- Applied stress

$B$ - Width of the footing

$E_{s}$ - Elastic modulus of soil below the footing

$\mu$ - Poisson ratio of the soil

$\mu_{0}, \mu_{1}$ - Factors depending on width of the footing, depth of the footing and thickness of the compressible medium. (Bowles, 1988).

\subsubsection{Method using elastic theory (Bowels, 1988)}

Settlement $(\rho)$ is estimated using the Equation [2] given below.

$\rho=q B\left(\frac{1-\mu^{2}}{E_{s}}\right) I_{n}$

Where symbols have their usual meaning and $I_{w} \quad$ - Settlement influence factor (Bowles, 1988). 
In this paper, this method will be referred to as the Elastic method_2.

\subsubsection{Method proposed by Timoshenko and Goodier (1951) as presented by Bowles (1997)}

Settlement $(\rho)$ is estimated using Equation [3] given below.

$\rho=q B^{\prime}\left(\frac{1-\mu^{2}}{E_{s}}\right) m I_{s} I_{F}$

Where symbols have their usual meaning and $\mathrm{M}$ - for settlement at the centre of a rectangular area $\mathrm{m}=4$

B/ - For the settlement at he centre B/ = B/2

$I_{S}, I_{F}$ - Settlement influence factor and depth factor (Bowles, 1997)

In this paper, this method will be referred to as the Elastic method_3.

\subsection{Settlement estimation using empirical correlations}

There are large number of empirical correlations to estimate the settlement of footings using insitu test results such as SPT and CPT. Due to the wide usage, the method proposed by Terzaghi and Peck (1967) is considered here. According to the Terzaghi and Peck (1967) method, the settlement is given by Equation [4].

$$
\rho=\frac{3 q}{N}\left[\frac{2 B}{B+1}\right]^{2}
$$

Where

$\rho=$ Settlement of foundation in inches

$B=$ Width of the foundation in feet

$\mathrm{q}=$ Applied bearing pressure in tons $/ \mathrm{ft}^{2}$

$\mathrm{N}=$ Average SPT value over a depth of $2 \mathrm{~B}$

\subsection{Settlement estimation using plate load test results}

The plate load tests are conducted according to the AASHTO T - 235 - 74, ASTM D 1194 - 72, or any other specification. Generally a circular or square plate of $300 \mathrm{~mm}$ or $450 \mathrm{~mm}$ is loaded in stages until the stress applied on the soil reaches a pre-determine level or upto the failure of the soil underneath. During each stage of the loading the settlement of the plate is monitored with time, while the load applied on the plate maintained constant, until the rate of the settlement of the plate is smaller than a specified value or a certain specified time duration lapses. The settlement at the end of each stress increment is plotted against the corresponding stress applied to obtain the settlement vs. applied stress curve, commonly referred to as the 'load - settlement' curve.

As the saturated clayey soils undergo time dependent consolidation settlement, when subjected to external stresses, it is very difficult to achieve the low level of settlement rate as specified in the test procedure. Therefore, use of the plate load test is highly questionable in places, where saturated clayey soil layers are present near the ground surface.

As previously mentioned, the settlement of the footings are estimated using extrapolation techniques. The extrapolation techniques are developed mostly using settlement estimation methods by determining the unknown soil parameters using the load - settlement behavior obtained from the plate load test. However, these extrapolation methods assume that the soil properties below the plate are homogeneous and isotropic to a considerable depth, at least upto the influence zone of the footing.

Figure 1 shows the influence zones of the plate and a prototype footing in a layered soil medium with a strong soil overlying a weak soil layer. It is clear from the depth of penetration of the influence zones, the settlement of the plate is not affected by the weak layer but the settlement of the actual footing is influence by the weak layer, as the influence zone of the footing extends well into the weak layer. Therefore, the settlement extrapolation methods may under predict the settlement of the actual footing.

\subsubsection{Settlement extrapolation based on the Terzahgi and Peck method}

Based on the settlement estimation method given as Equation [4], Terzaghi and Peck (1967) proposed the following settlement extrapolation relationship, given below as Equation [5], for the plate load test results.

$\rho_{\text {footing }}=\rho_{\text {Plate }}\left(\frac{2 B_{\text {Footing }}}{B_{\text {Footing }}+B_{\text {Plate }}}\right)^{2}$

Where

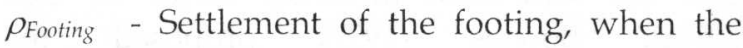
applied stress is $q$

Pplate - Settlement of the plate, when the applied stress is $q$

$B_{\text {Plate }}$ - Diameter of the plate

$B_{\text {Footing - Diameter of the footing }}$ 


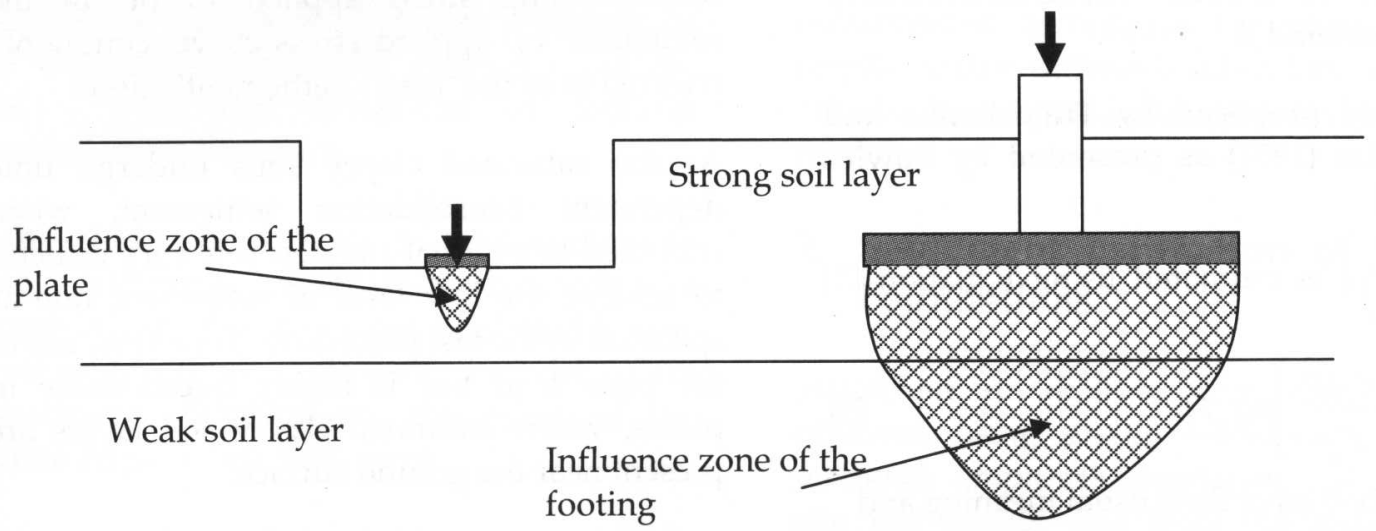

Figure 1 - Influence zones of the plate and the footing in a layered medium with a strong soil overlying a weak soil layer.

For example, if the settlement of the foundation has to be estimated at a certain stress, say 100 $\mathrm{kPa}$, the settlement of the plate at $100 \mathrm{kPa}, \rho_{\text {Plates }}$ is obtained from the load - settlement curve and substituted into Equation [5]. Knowing the dimensions of the plate and the foundations, the settlement of the foundation ( $\left.\rho_{\text {footing }}\right)$ may be estimated.

\subsubsection{Settlement extrapolation based on Janbu method}

Using Equation [1], the settlement of the footing may be obtained using the Equation [6] given below.

$$
\rho_{\text {Footing }}=\rho_{\text {Plate }}\left(\frac{\left(\mu_{1} \mu_{2} B\right)_{\text {Footing }}}{\left(\mu_{1} \mu_{2} B\right)_{\text {Plate }}}\right)
$$

\subsubsection{Settlement extrapolation using Elastic method_2}

Using Equation [2], the settlement of the footing may be obtained using the Equation [7] given below.

$\rho_{\text {Footing }}=\rho_{\text {Plate }}\left(\frac{\left(I_{w} B\right)_{\text {Footing }}}{\left(I_{w} B\right)_{\text {Plate }}}\right)$

\subsubsection{Settlement extrapolation using Elastic Method 3}

Based on the settlement estimation equation proposed by Timoshenko and Goodier (1951) and the settlement influence factor by Fox (1948), Bowles (1997) proposed the extrapolation relationship given in Equation [8] for the plate load test results.

$$
\frac{\rho_{\text {Footing }}}{\rho_{\text {plate }}}=\frac{q_{o 2} B_{2}^{\prime} m I_{s 2} I_{F 2} E_{s 2}^{\prime}}{q_{o 1} B_{1}^{\prime} m I_{s 1} I_{F 1} E_{s 1}^{\prime}}
$$

If the settlement under the same contact pressure for both plate and the footing are considered, Equation [8] can be modified to a rectangular footing as given in Equation [9].

$$
\frac{\rho_{\text {Footing }}}{\rho_{\text {plate }}}=\frac{B_{\text {Footing }} I_{\text {Footing }} I_{\text {FFooting }} E_{\text {Footing }}}{B_{\text {plate }} I_{\text {FPlate }} E_{\text {Plate }}}
$$

Where

$I_{\text {Footing, }} I_{\text {Plate }} \quad$ - Settlement influence factor for the footing and plate (Bowles, 1997).

$I_{\text {FFooting }} I_{\text {FPlate }}$ - Depth correction factor for the footing and plate (Bowles, 1997).

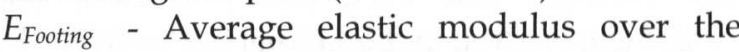
effective depth $H\left(=5 B_{\text {Footing }}\right.$ or actual depth to the hard stratum).

$E_{\text {Plate }}$ - Average elastic modulus over the effective depth $H$ ( $=5 B_{\text {Plate }}$ or actual depth to the hard stratum).

\section{Methodology Adopted in the Investigation}

It is intended to investigate the accuracy of the settlement estimation methods discussed above. Ideally such comparisons should be carried out by comparing the settlement predicted from different methods with the corresponding settlement measured in fullscale field load testing of the shallow foundations. However, conducting such fullscale load testing is not feasible in a developing country like Sri Lanka. Therefore, other lowcost methods should be used to obtain such data. The method used should be reliable and verified against some field test results.

Finite element (FE) models are very widely used for simulation of similar field problems. However, FE models, or any other numerical 
simulation tools, should be used with caution as there are certain limitations on these numerical simulation techniques. The verification of the results obtained from such numerical tools is an essential part of any such study using them. The verification may come from comparing the results of numerical models with the relevant field measurements and/or by investigating the variability of the model predictions with the established engineering principles of the relevant parameters.

In this study, a FE model is developed to simulate the behavior of a loaded shallow foundation. The model predictions are verified using some field full-scale results available in the literature. Moreover, variability of the model predictions are also compared with the existing accepted knowledge of the relevant parameters.

\subsection{Finite element model}

A popular finite element software, referred to as PLAXIS used for problems associated with geotechnical engineering, is used for the simulation of the plate load test and the loading of the actual footings. The Mohr-Coulomb failure criterion with the linear elastic behavior inside the yield surface and elasto-plastic behavior with the associated flow rule is used as the soil constitutive model. Considering the accuracy and the economy of the solution process, axisymmetrical modeling was carried out and the mesh configuration was selected after carrying out a sensitivity analysis.
The soil is modelled using fifteen-node isoparametric elements with the soil constitutive model mentioned above. The plate or the footing is simulated with plate elements with linear elastic constitutive behavior. The interface between the foundation and the soil underneath was assumed to show full frictional behavior. The ultimate carrying capacity prediction of the model was not satisfactory due to the numerical instability at the higher strain levels. However, model predictions under low strain levels corresponding to the generally observed working stress levels were highly satisfactory.

\subsection{Comparison of the FE model predictions with field measurements}

Full-scale testing of shallow foundations, reported in D'Appolonia et al. (1968) and Schmertmann (1970) were modeled using the developed FEM model. The reported full-scale tests were carried out by loading rectangular footings with length to width ratios varying from 1.0 to 8.8. Since it is not possible to develop a 3-dimensional model with the available computer resources, axisymmetrical and plane-strain modelling of the reported cases was carried out. Footings with length to width ratio close to unity were modelled as axisymmtrical with the equivalent area and the footings with higher length to width ratios were modelled as strip footings with plane-strain behaviour. The measured settlements of the footings and the settlement predicted by the developed FE model are given in Table1.

Table 1 - Measured settlement and the settlement predicted by the developed FE model for some case studies presented in the literature.

\begin{tabular}{|c|c|c|c|c|c|c|c|c|}
\hline \multirow[t]{2}{*}{ reference } & \multirow[t]{2}{*}{$\mathrm{H} / \mathrm{B}$} & \multirow[t]{2}{*}{$\mathrm{B}(\mathrm{m})$} & \multirow[t]{2}{*}{$\mathrm{L} / \mathrm{B}$} & \multirow{2}{*}{$\begin{array}{l}\mathrm{E}_{\mathrm{s}} \\
(\mathrm{kPa})\end{array}$} & \multirow[t]{2}{*}{$\mu$} & \multirow{2}{*}{$\begin{array}{l}\Delta \sigma \\
(\mathrm{kPa})\end{array}$} & \multicolumn{2}{|c|}{ Settlement (mm) } \\
\hline & & & & & & & Measured & Estimated \\
\hline $\begin{array}{l}\text { D'Appolonia } \\
\text { et al. (1968) }\end{array}$ & 4 & 3.8 & 1.6 & 57456 & 0.33 & 163 & $7.6-10.2$ & 14 \\
\hline \multicolumn{9}{|c|}{ Schmertmann (1970) } \\
\hline Case 1 & 5 & 2.6 & 8.8 & 14843 & 0.4 & 179 & 38.86 & 41.5 \\
\hline Case 2 & 5 & 3.0 & 4.2 & 29686 & 0.3 & 160 & $20.3-22.9$ & 26.5 \\
\hline Case 5 & 5 & 18.9 & 1.0 & 16758 & 0.45 & 75 & 63 & 68.5 \\
\hline Case 6 & 1 & 26.5 & 2.2 & 11012 & 0.3 & 198 & 269 & 336 \\
\hline Case 8 & 5 & 0.60 & 1.0 & 5267 & 0.3 & 109 & 6.86 & 14.8 \\
\hline
\end{tabular}

The measured and the estimated settlements agree reasonably well except for the last two cases, where the FEM method slightly overestimates the settlements. It should be noted here that the modelling was done assuming plane strain or axisymmetrical conditions even though actual footings are rectangular. The predictions for footings having higher L/B ratios are better compared to other cases.

The model predictions were also checked against the accepted variations of the settlement 
of shallow foundations. For example, the variation of the settlement of the footings with the foundation depths and the variation of the settlement of footings with the footing widths were investigated. It was observed that the settlement predictions of the model were in accordance with the accepted variations.

\section{Parametric Study}

\subsection{Estimation of the settlement of shallow foundations}

Settlement of shallow foundations are very often estimated using the elastic theories. Even the empirical correlations used in the settlement estimation assume that the settlement is linearly proportional to the applied pressure (eg, Equation [4]). However, it is commonly accepted that the soil shows nonlinear behavior even under small stress levels. To investigate the accuracy of the three settlement prediction methods given in section $2.1,1 \mathrm{~m}, 2 \mathrm{~m}$ and $5 \mathrm{~m}$ diameter foundations, placed at a depth of $0.9 \mathrm{~m}$ below the ground surface are considered. Three foundations of diameter $1 \mathrm{~m}, 2 \mathrm{~m}$ and $5 \mathrm{~m}$ are placed in soil mediums having different properties. The settlement predicted by the elastic settlement estimation methods were compared with the settlement predicted by the developed FE model. The settlement of the footings and the settlement estimated by the elastic settlement estimation methods for two of the isotropic homogeneous sandy soil mediums considered in the study, one soil type (Soil A) having properties $\mathrm{E}=20000 \mathrm{kPa}, \phi=33^{\circ}$, poisson ratio 0.25 and the other soil type (Soil B) having $\mathrm{E}=80000 \mathrm{kPa}, \phi=40 \mathrm{o}$, poisson ratio 0.25 , are presented here. The load-settlement curves obtained for different size footings in the two soil mediums mentioned above are shown in Figures 2 and 3 respectively.

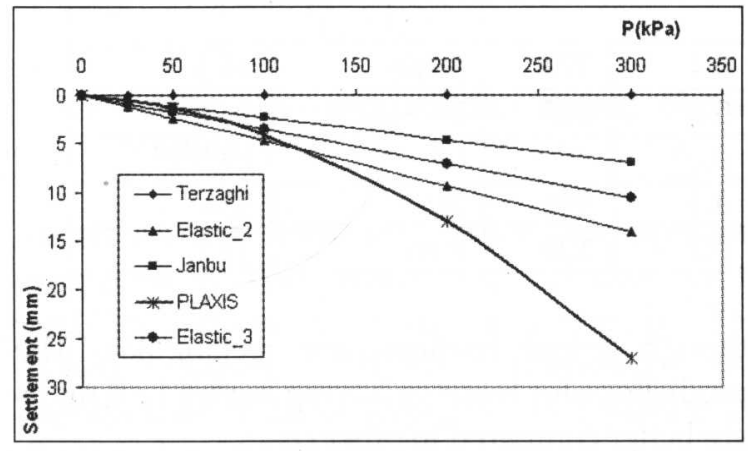

(a) $1 \mathrm{~m}$ diameter footing

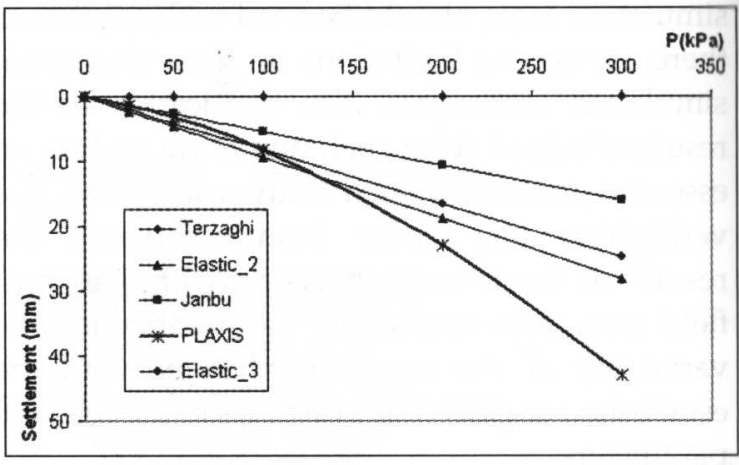

(b) $2 \mathrm{~m}$ diameter footing

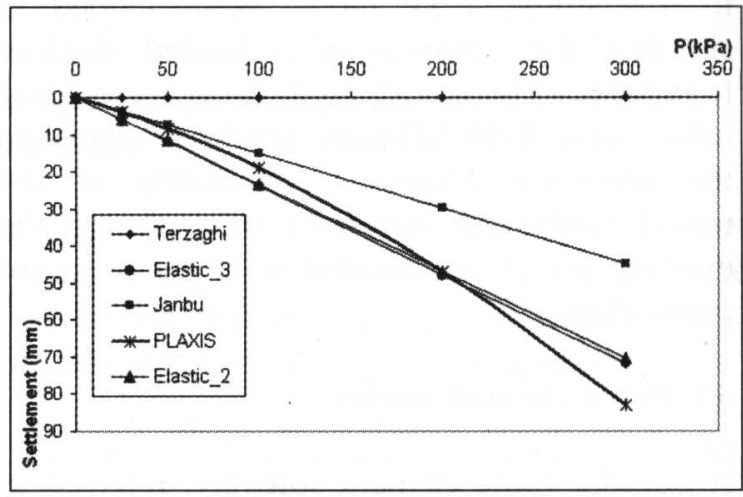

(c) $5 \mathrm{~m}$ diameter footing

Figure 2:Load - settlement curves estimated for footings in Soil A with $\phi=33^{\circ}$ and $E=$ $20000 \mathrm{kPa}$.

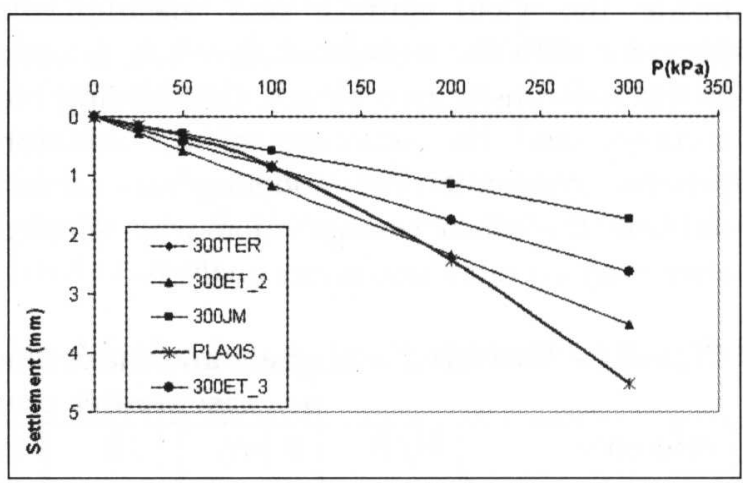

(a) $1 \mathrm{~m}$ diameter footing

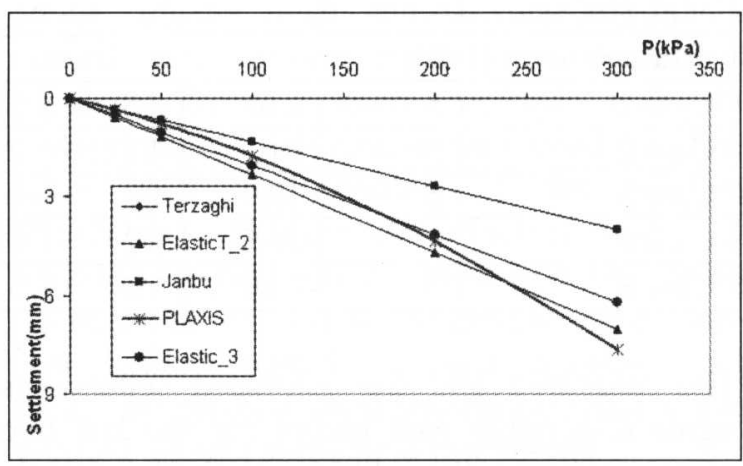

(b) $2 \mathrm{~m}$ diameter footing 


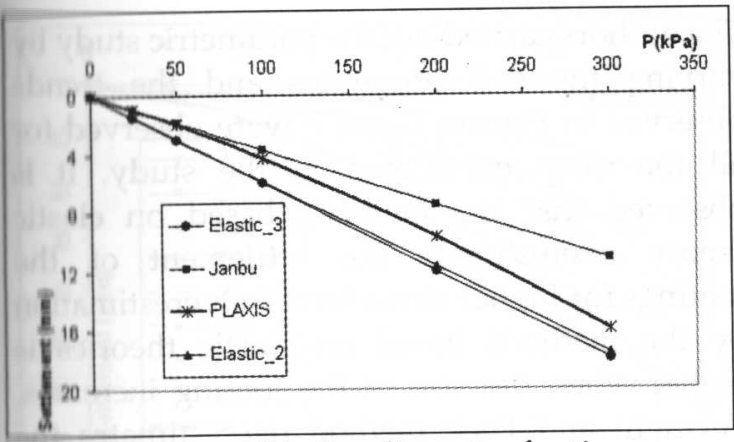

(b) 3m diameter footing

\section{Figure 3:Load - settlement curves estimated for footings in Soil B with $\phi=40^{\circ}$ and $E=$ $80000 \mathrm{kPa}$.}

The variations of the settlement estimated in Figures 2 and 3 indicate general trend shown in the parametric study. As shown in Figures 2 and 3 , the settlement estimated by the elastic methods highly underestimates the settlement of footings for smaller size footings in 'weaker' soil layers at higher stress levels. However, when the footing size increases and/or the soil becomes stiffer, the accuracy of the settlement estimated from the elastic methods, were substantially improved. Out of the elastic settlement estimation methods considered in this study, settlements estimated from the Janbu method always underpredicted the settlement. Bowles (1996) stated that the elastic method_3 is one of the best elastic settlement estimation methods available. However, the present study reveals that the settlements predicted by the elastic method_2 are slightly. better than the predictions from the elastic method_3 for all the cases considered. Elastic method_2 and Elastic method_3 are explained in sections 2.1.2 and 2.1.3 respectively.

\subsection{Extrapolation of the plate load test results in homogeneous medium}

As explained earlier, settlements obtained from loading a smaller size plate should be extrapolated to large size foundations. To investigate the accuracy of the extrapolation techniques explained in section 2.3 , both plate load test results and the actual prototype foundation behavior are determined using the FEM. The two soil types considered in section 4.1 , Soil $\mathrm{A}$ having properties $\mathrm{E}=20000 \mathrm{kPa}, \phi=$ $33^{\circ}$, poisson ratio 0.25 and Soil $\mathrm{B}$ having $\mathrm{E}=$ $80000 \mathrm{kPa}, \phi=40^{\circ}$, Poisson ratio 0.25 were considered for this study. Load - settlement behavior of two $300 \mathrm{~mm}$ and $450 \mathrm{~mm}$ diameter circular plates in Soil A and Soil B were obtained from the developed FE simulation model. The simulated plate load test results, thus obtained, were extrapolated to $1 \mathrm{~m}, 2 \mathrm{~m}$ and $5 \mathrm{~m}$ diameter footings at $0.9 \mathrm{~m}$ below the ground surface. The extrapolated load - settlement behavior of different sizes of footings and the FEM predicted load-settlement behavior of the corresponding footings in Soil A and Soil B are shown in Figures 4 and 5 respectively.

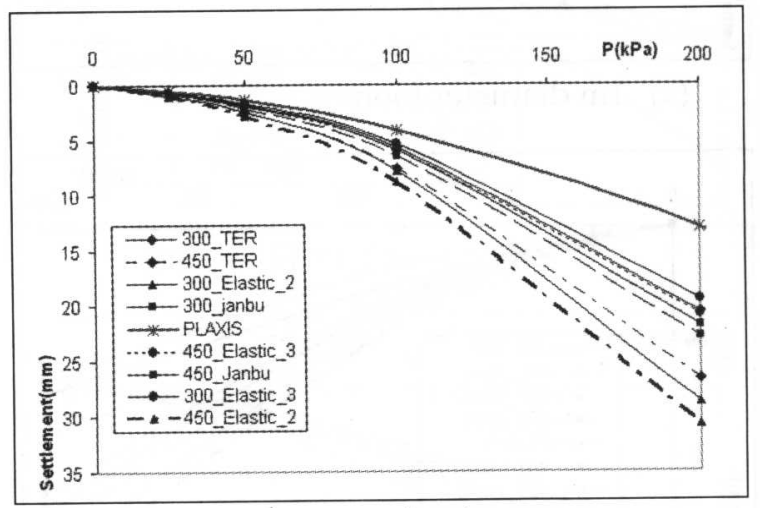

(a) $1 \mathrm{~m}$ diameter footing

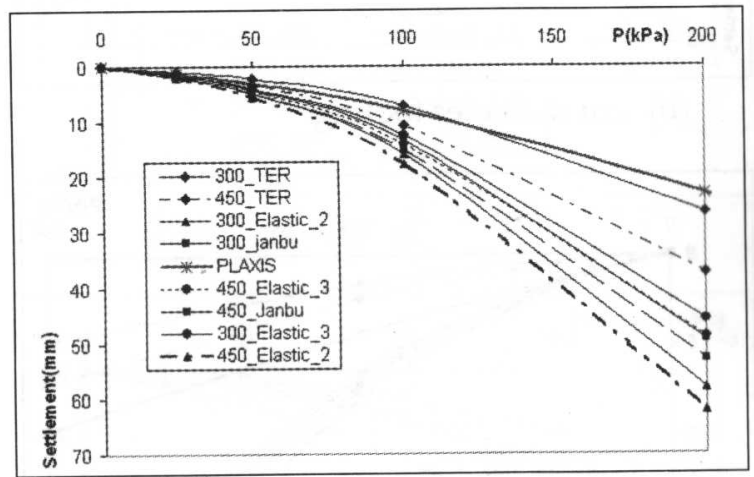

(b) $2 \mathrm{~m}$ diameter footing

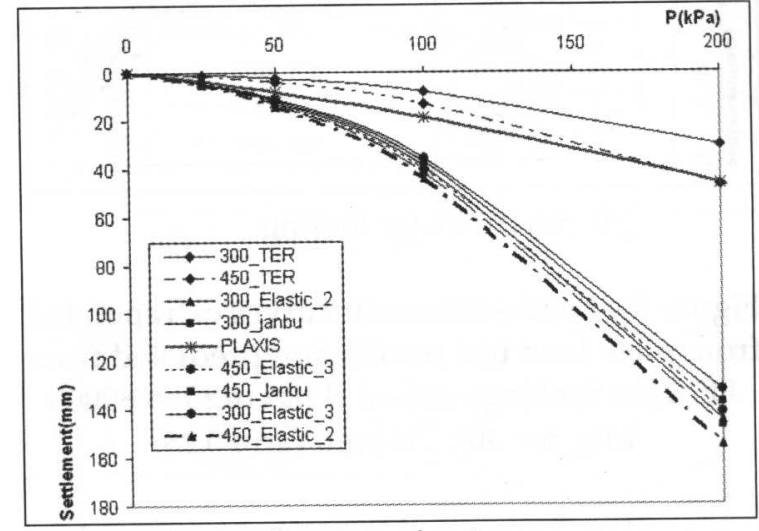

(c) $5 \mathrm{~m}$ diameter footing

Figure 4 - Load-settlement curves extrapolated from plate load test results and predicted from FEM for footings in Soil A having $E=20000$ $\mathrm{kPa}, \phi=33^{\circ}$, Poisson ratio 0.25 . 


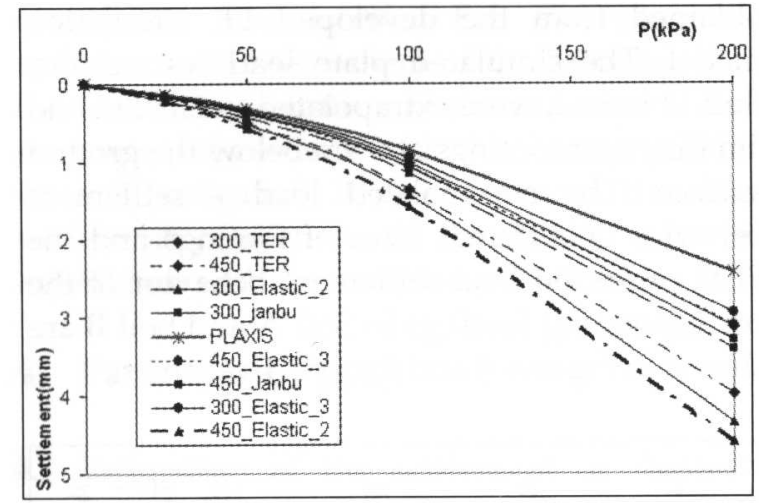

(a) $1 \mathrm{~m}$ diameter footing

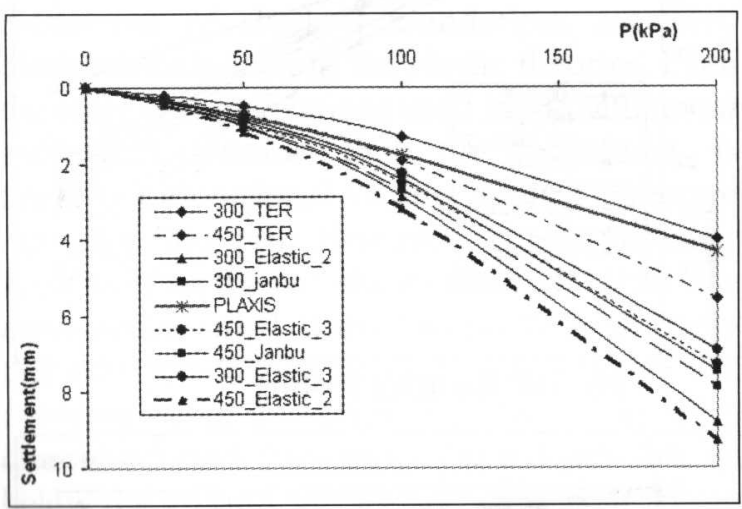

(b) $2 \mathrm{~m}$ diameter footing

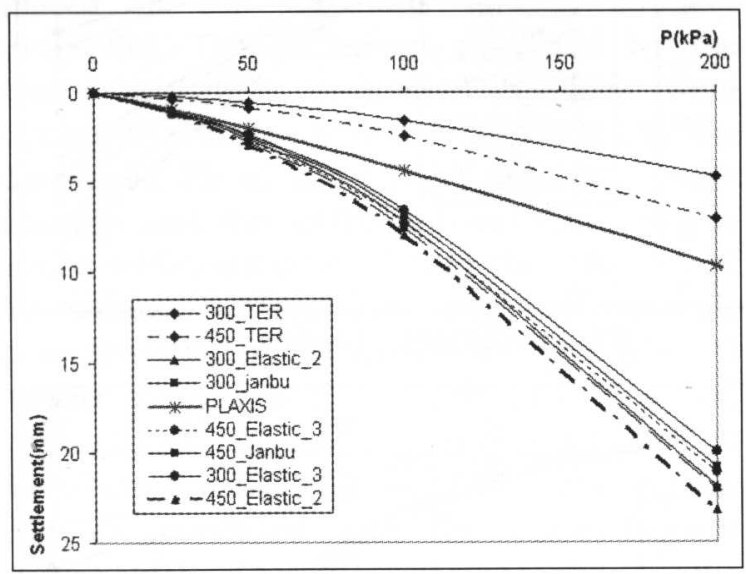

(d) $5 \mathrm{~m}$ diameter footing

Figure 5 - Load-settlement curves extrapolated from plate load test results and predicted from FEM for footings in Soil $B$ having $E=80000$ $\mathrm{kPa}, \phi=40^{\circ}$, Poisson ratio 0.25.
The authors carried out the parametric study by varying the soil properties and the trends observed in Figures 6 and 7 were observed for all the cases considered in the study. It is observed that the methods based on elastic theory overestimate the settlement of the footings for higher stress levels. Overestimation by the methods based on elastic theories is higher when the size of the footing increases. Terzaghi and Peck method overestimates the settlement of smaller size footings while underestimates the settlement of the larger footings. However, error associated with the Terzaghi and Peck method is the least compared to the other extrapolation methods.

\subsection{Extrapolation of the plate load test results in the layered medium}

A parametric study was carried out to investigate the effects of soil layering on the extrapolation of the plate load test results. Layered soil mediums with soft layer underlying a strong soil layer, as shown in Figure 6, were considered, as there is a possibility of underestimation of the settlement in such situations. A parametric study was carried out to investigate the effects of the top layer thickness on the extrapolation of the plate load test results. One such parametric study with stronger soil (Soil 1) having $E_{1}=24000$ $\mathrm{kPa}, \phi_{1}=33^{\circ}, \mu_{1}=0.25$ and the weaker soil (Soil 2) having $E_{2}=6000 \mathrm{kPa}, \phi_{2}=25^{\circ}$ and $\mu_{2}=0.25$, as given in Figure 6, are presented here. The thickness of the top layer below the foundation level $(H)$ was varied to $0.5 \mathrm{~m}, 1.0 \mathrm{~m}, 2.0 \mathrm{~m}$ and $4.0 \mathrm{~m}$. Plate load tests were simulated for different $\mathrm{H}$ values and the results were extrapolated to a $2 \mathrm{~m}$ diameter footing assuming homogeneous soil conditions below the foundation level. The settlement of the footing in the layered soil medium was also estimated by the FE model developed. In Figure 7, the load-settlement curves obtained from extrapolated plate load test results for different $\mathrm{H}$ are compared with the corresponding loadsettlement curves obtained from the FE model.

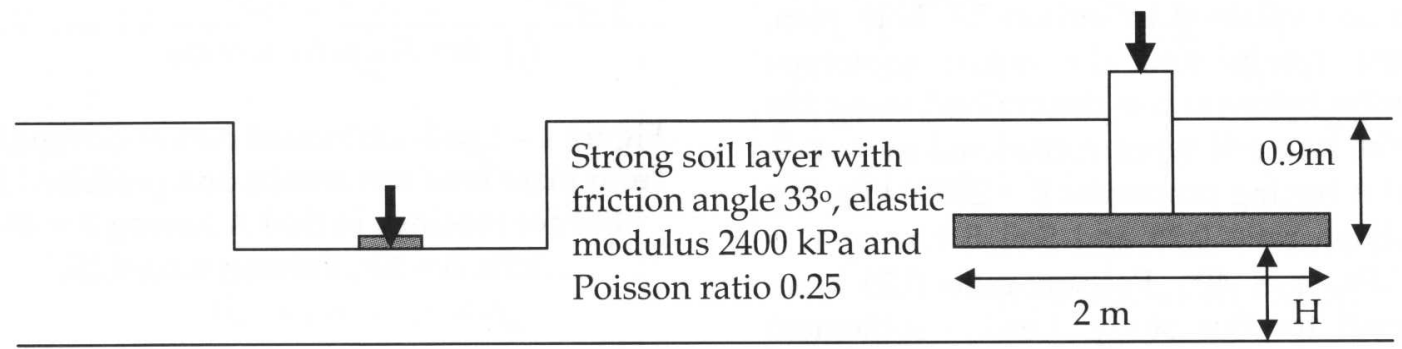

Soft soil laver with friction angle $25^{\circ}$, elastic modulus $6000 \mathrm{kPa}$ and Poisson ratio 0.25

Figure 6 - Cross sectional view of the layered soil medium. 


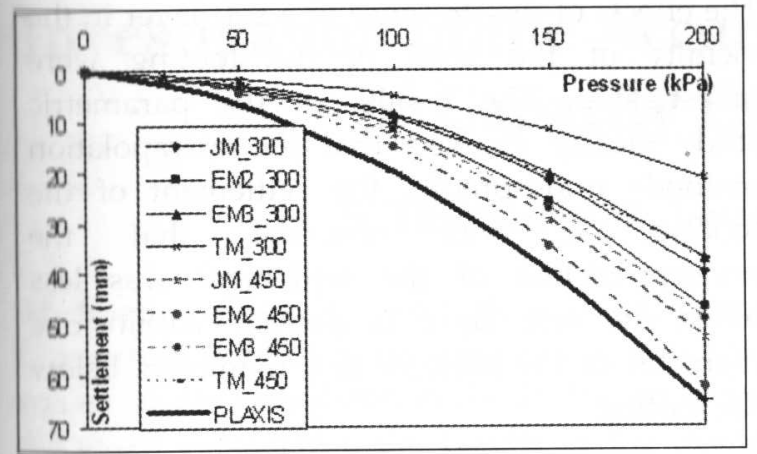

(a) $\mathrm{H}=0.5 \mathrm{~m}$

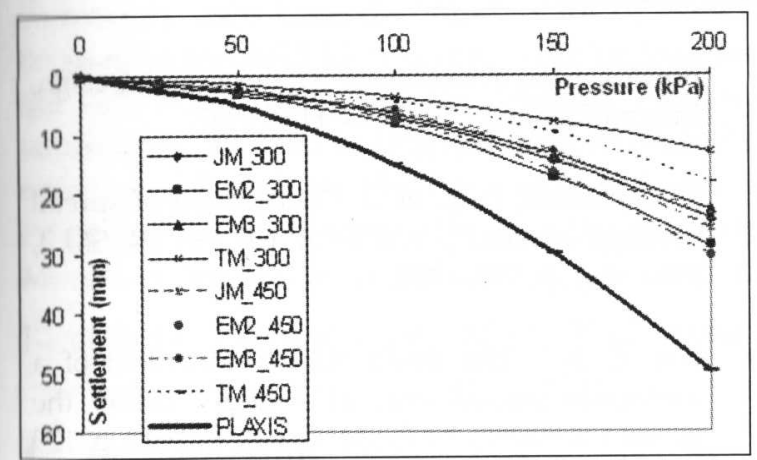

(b) $\quad \mathrm{H}=1.0 \mathrm{~m}$

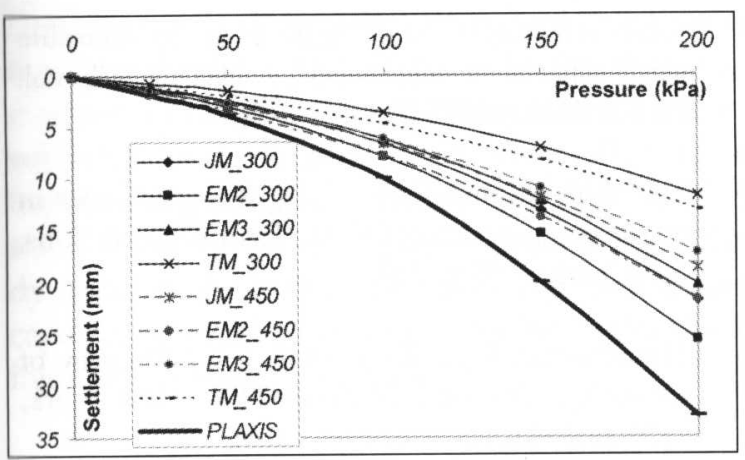

(c) $\mathrm{H}=2.0 \mathrm{~m}$

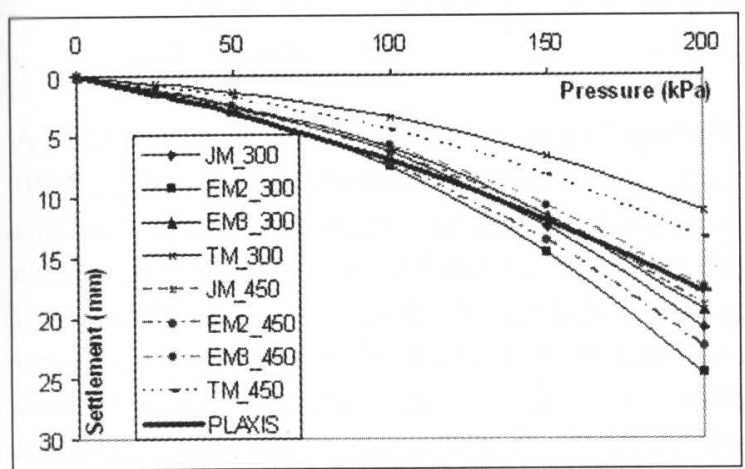

(d) $\quad \mathrm{H}=4.0 \mathrm{~m}$

Figure 7 - Load - settlement curves for different values of $H$ from extrapolation of plate load test results and FE model for a $2 \mathrm{~m}$ diameter footing in the layered soil medium shown in Figure 6.

The settlement estimated by the different extrapolation methods minus the settlement from the FE model are presented in Figure 8 for different $H$ values. The negative values indicate underestimation of the settlement of the footing and positive values indicate overestimation of the settlement of the footing.

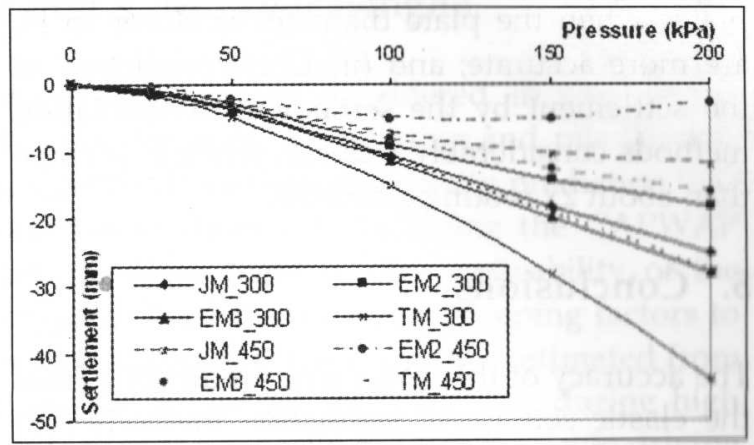

(a) $\mathrm{H}=0.5 \mathrm{~m}$

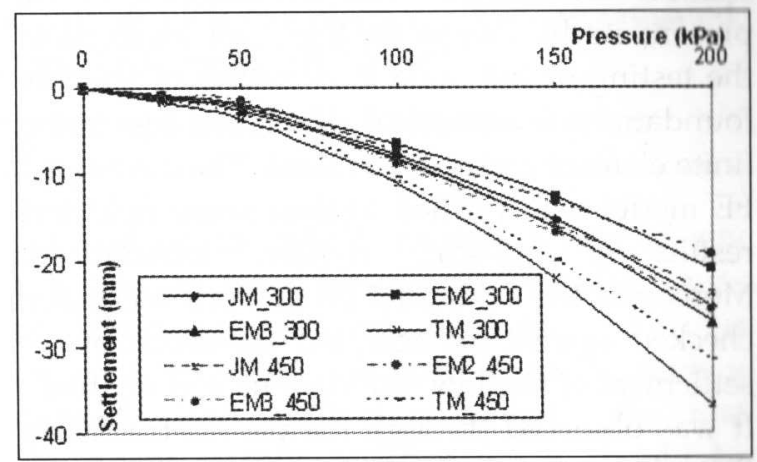

(b) $\mathrm{H}=1.0 \mathrm{~m}$

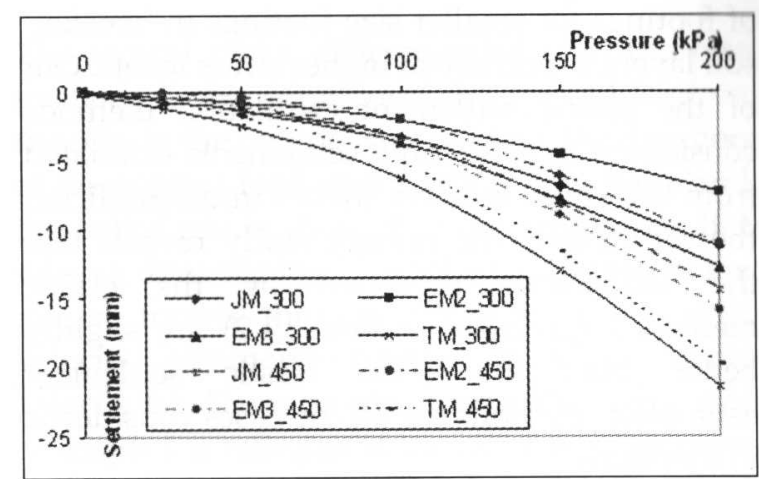

(d) $\mathrm{H}=2.0 \mathrm{~m}$

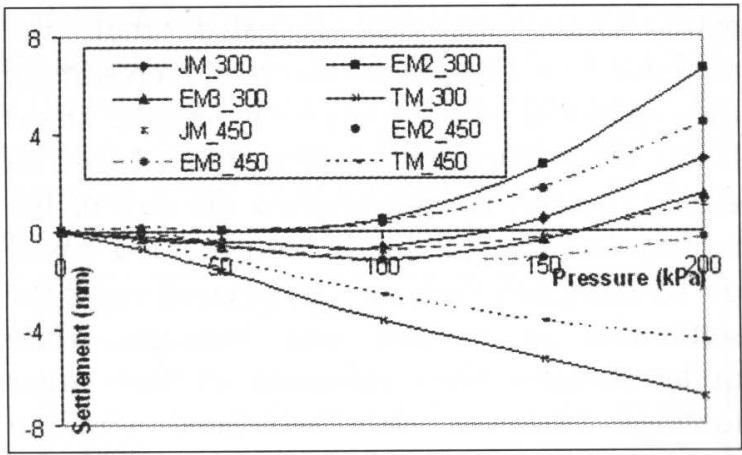

(e) $\mathrm{H}=4.0 \mathrm{~m}$

Figure 8 - The settlement estimated by the extrapolation methods minus the settlement from the FE model for different $\mathrm{H}$ values. 
The results of the parametric study clearly showed that the extrapolation methods underpredict the settlement of the footing as expected. However, there are two points to be observed: (i) the settlements, predicted by the plates when the plate diameter is closer to $\mathrm{H}$, are more accurate; and (ii) Underprediction of the settlement by the settlement extrapolation methods considerably reduces, when $H$ is more than about $2 x$ footing diameter.

\section{Conclusions}

The accuracy of the settlement predictions from the elastic settlement estimation methods and extrapolation of the plate load test results of shallow foundations were investigated in the present study. Due to the high cost involved in the testing of full scale load testing of shallow foundations, a numerical simulation tool using finite element method was used. The developed FE model was verified against some field test results of full-scale shallow foundations. Moreover, the FE model predictions were also checked against the accepted variations of the settlement of shallow foundations and verified. It was observed through the parametric study that the settlement estimated by the elastic methods highly underestimates the settlement of footings for smaller size footings in 'weaker' soil låyers, especially at higher stress levels. Out of the elastic settlement estimation methods considered in this study, settlements estimated from the Janbu method always underpredicted the settlement. The present study reveals that the settlements predicted by the elastic method_2 (given in section 2.1.2) are slightly better than the other elastic settlement estimation methods for all the cases considered in this study.

The investigation of the settlement extrapolation methods revealed that the methods based on elastic theories overestimate the settlement of the footings for higher stress levels. Overestimation by the methods based on elastic theories is higher when the size of the footing increases. Extrapolation method based on Terzaghi and Peck method overestimates the settlement of smaller size footings while underestimates the settlement of the larger footings. However, error associated with the extrapolation method Terzaghi and Peck method is the least compared to other methods.
The effects of the presence of a soft layer in the vicinity of the level of the footing were investigated. The results of the parametric study clearly showed that the extrapolation methods underpredict the settlement of the footing. It was observed that the underprediction of the settlement was less when the soft layer is present within one diameter of the plate or two diameters below the footing.

\section{References}

1 Bowles, J. E., Foundation Analysis and Design, International ed., McGraw-Hill, 1997.

2 D'Appolonia, D. J., et al., "Settlement of spread footings on sand", JSMFD, ASCE, vol. 94, SM 3, 1968 May, p. 735 - 760.

3 Fox, E. N., "The mean elastic settlement of a uniformly loaded area at a depth below the ground surface", 2 ${ }^{\text {nd }}$ ICSMFE, vol. 1, 1948, p. 129 $-132$

4 Schmertmann, J. H., "Static cone to compute static settlement, over sand", JSMFD, ASCE, vol. 96, SM 3, May 1970. Pp. $1011-1043$.

5 Terzaghi, K., and Peck, R. B., Soil mechanics in engineering practice, $2^{\text {nd }}$ ed., John Wiley \& Sons, New York, 1967 p. 729.

6 Timoshenko, S., and Goodier, J. N., Theorey of Elasticity, 2nd ed., McGraw-Hill, New York, 1951, p. 580. 